\title{
Integrated MEMS Hydrogen Gas Sensor with PdCuSi-based Hydrogen Actuators and Micro Thermal Conductivity Detector
}

\author{
Hiroaki Yamazaki ${ }^{1}$, Yumi Hayashi', Kei Masunishi², Daiki Ono ${ }^{1}$, Tomohiro Saito ${ }^{1}$, Akihiro Kojima1 \\ ${ }^{1}$ Corporate Research and Development Center, Toshiba Corporation, Kawasaki, Japan \\ ${ }^{2}$ Corporate Manufacturing Engineering Center, Toshiba Corporation, Yokohama, Japan \\ hiroaki.yamazaki@toshiba.co.jp
}

\begin{abstract}
:
We report a dual-range MEMS hydrogen sensor that has a micro thermal conductivity detector ( $\mu$ TCD) and a capacitive sensor with PdCuSi-based hydrogen actuators. A surface micromachining process with double TiN layers is proposed for integration of the capacitive sensor and the $\mu$-TCD on the same chip. We confirmed that both of the fabricated sensors exhibited a fast response with no hysteresis. The capacitive sensor had high sensitivity for low hydrogen concentrations ranging from $0.05 \mathrm{vol} \%$ (500 ppm) to $5 \mathrm{vol} \%$ despite being heater-less, while the $\mu$-TCD had excellent linearity for high concentrations near 100 vol \%. Furthermore, the capacitive sensor showed excellent selectivity for hydrogen thanks to the material properties of $\mathrm{PdCuSi}$. Therefore, the proposed sensor is suitable for hydrogen leakage detection with low power consumption and for precise measurement of highconcentration hydrogen gas.
\end{abstract}

Key words: Hydrogen sensor, MEMS, PdCuSi metallic glass, thermal conductivity detector, capacitive sensor

\section{Background}

In the future hydrogen society, hydrogen leakage sensors with high sensitivity and low power consumption will be indispensable to ensure safety. Meanwhile, a precise concentration meter is also necessary for measuring hydrogen gas concentrations in hydrogen gas process lines. In this paper, we propose a novel integrated MEMS hydrogen sensor that realizes both of leak detection and measurement of hydrogen gas.

\section{Description of the Proposed Sensor}

Fig. 1 shows a cross-sectional image of the proposed integrated MEMS hydrogen sensor. We integrated the $\mu$-TCD and the capacitive sensor with PdCuSi-based hydrogen actuators on a single chip.

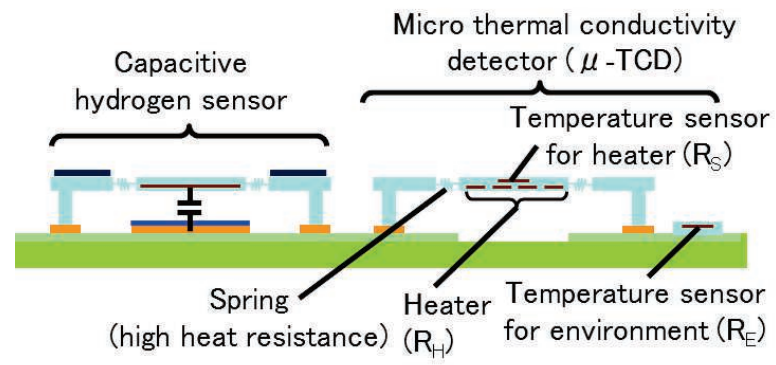

Fig. 1. Proposed Integrated MEMS hydrogen sensor
The capacitive sensor has a PdCuSi-based hydrogen actuators, a movable membrane with an embedded electrode, and a fixed electrode as shown in Fig. 2. The hydrogen actuators are connected to the movable membrane via a spring. Unlike pure $\mathrm{Pd}, \mathrm{Pd}_{78} \mathrm{Cu}_{5} \mathrm{Si}_{17}$ has excellent properties as a hydrogen-sensing material, with a fast response and no hysteresis [1]. Hydrogen absorption in the PdCuSi film generates compressive stress. As a result, the hydrogen actuators mechanically move downward and the capacitance between the movable and fixed electrodes changes according to hydrogen concentration. As this operation occurs without heating, the sensing with low power consumption is realized. The $\mu$ TCD consists of an environment temperature sensor and a membrane that has a heater and temperature sensor (Fig.1). The $\mu$-TCD detects hydrogen by sensing the difference in thermal conductivity between air and hydrogen [2].

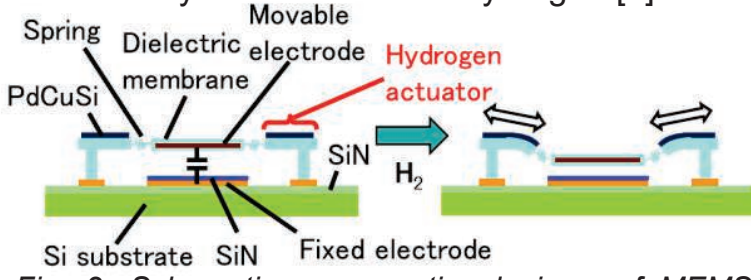

Fig. 2. Schematic cross-sectional views of MEMS capacitive hydrogen sensor with hydrogen actuators 


\section{Fabrication and Experimental Results}

The sensors were simultaneously fabricated using a surface micromachining process that is CMOS compatible (Fig. 3). We used a polyimide sacrificial layer and a plasma etching process in order to form mechanical structures. The heater and temperature sensor of the $\mu$ TCD is stacked in the membrane, resulting in realizing small size. The characteristics of the sensors were evaluated in a chamber in which gas composition could be changed. Fig. 4 shows response curves for 3 vol \% hydrogen gas in air. Both sensors rapidly responded to the hydrogen gas without hysteresis. The power consumption of $\mu$-TCD is $43 \mathrm{~mW}$. Furthermore, we confirmed the capacitive sensor can detect hydrogen of $0.05 \mathrm{vol} \%$ (500ppm) despite being heater-less.

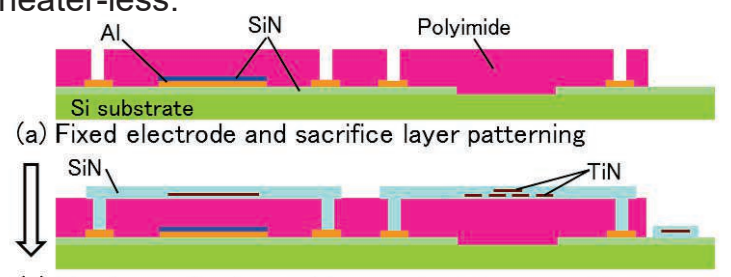

(b) Membrane, heater and temperature sensor patterning

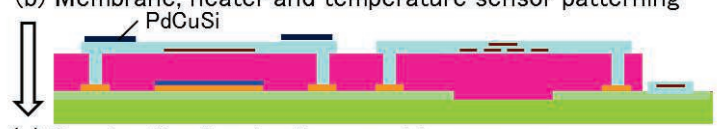

(c) Sensing film forming for capacitive sensor

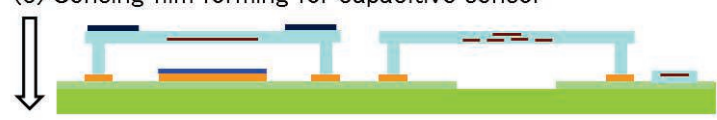

(d) Sacrifice layer removal by plasma etching

Fig. 3. Schematic process flow of the integrated MEMS sensor.
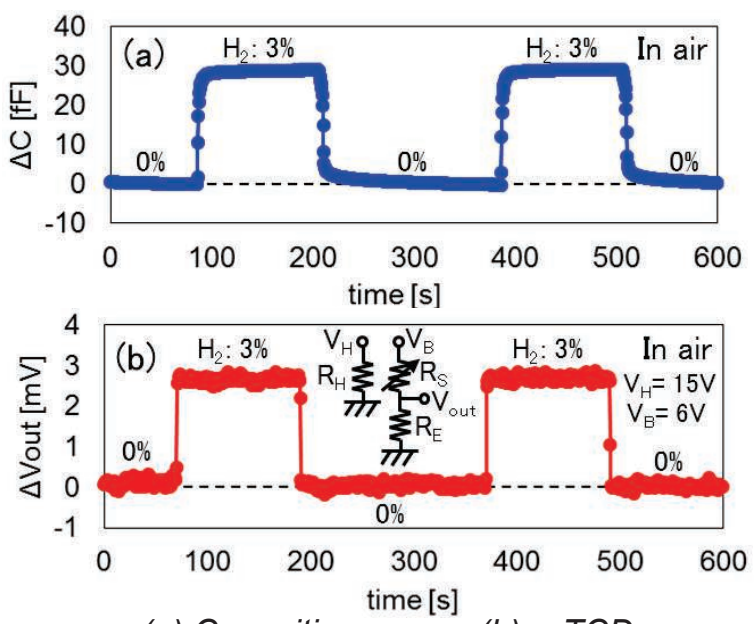

(a) Capacitive sensor (b) $\mu-T C D$

Fig. 4. Response characteristics to $\mathrm{H}_{2}$ gas.

We measured the dependence of sensor output on hydrogen gas concentration from 0 to 100 vol $\%$ as shown in Fig. 5. Nitrogen was used as the carrier gas. The sensitivity of the capacitive sensor was higher than that of the $\mu$-TCD in the low concentration range $(<5 \mathrm{vol} \%)$. On the other hand, the sensitivity of the $\mu$-TCD was higher than that of the capacitive sensor in the high concentration range. Moreover, the $\mu$-TCD showed excellent linearity in the measured concentration range (Fig. 6).

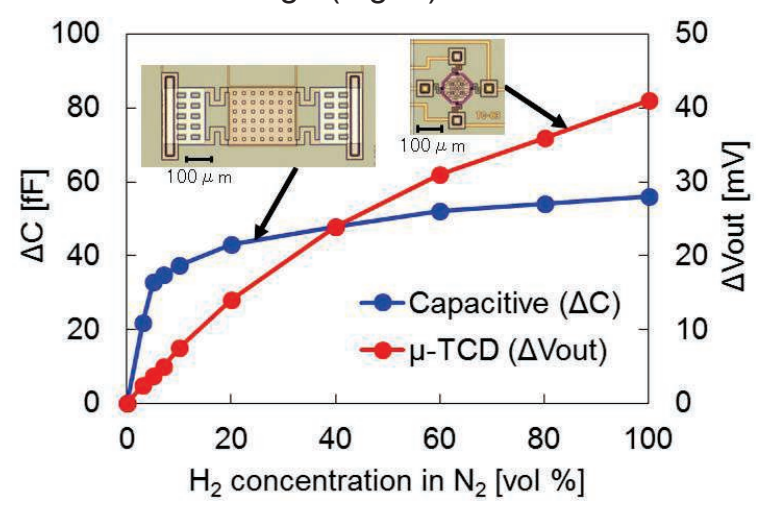

Fig 5. Comparison of $\mathrm{H}_{2}$ concentration dependence on sensor output for the capacitive sensor and the $\mu-T C D$.

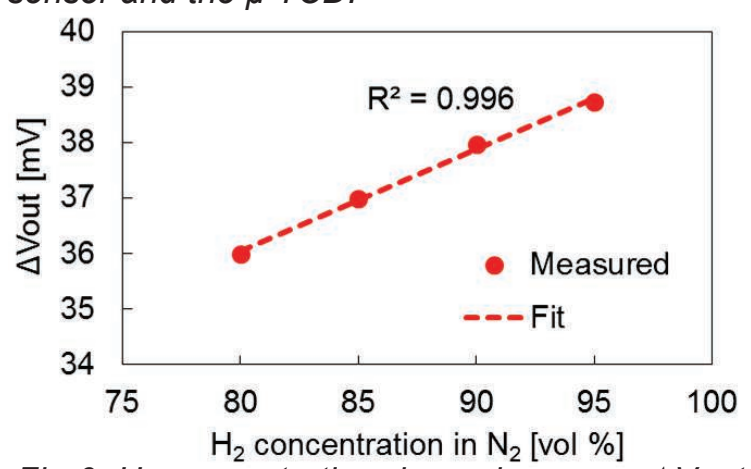

Fig 6. $\mathrm{H}_{2}$ concentration dependence on $\Delta$ Vout of $\mu-T C D$ for high concentration range.

Finally, we evaluated selectivity to hydrogen gas (Fig. 7). The $\mu$-TCD responds to also methane, carbon dioxide and helium gas according to their thermal conductivity. However, thanks to the properties of PdCuSi, the capacitive sensor did not respond gases other than hydrogen. Thus, the proposed sensor can achieve hydrogen leak detection with low power consumption, and precise measurement of high concentration hydrogen by utilizing each characteristics of both sensors.

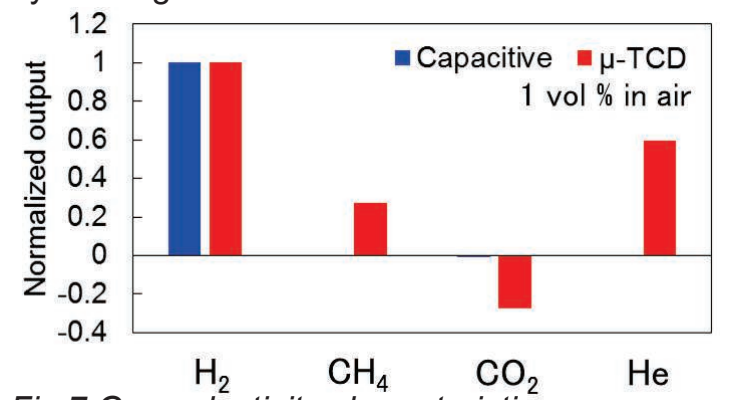

Fig. 7 Gas selectivity characteristics.

\section{References}

[1] Y. Hayashi et al, Transducers2017, 311-314

[2] I. Simon et al, Sensors and Actuators A 97-98, 104-108 (2002). 